\title{
Acer pentaphyllum - eine Rarität unter den Ahornen
}

\author{
VEIT MARTIN DÖRKEN
}

\begin{abstract}
Acer pentaphyllum is a small deciduous tree native to Sichuan in China. In its natural habitat it is highly endangered. There are less than 200 individuals growing in the wild. The species has a leaf shape that is unique within this group. The leaves are divided to the base and have 5 to 7 leaflets.
\end{abstract}

\section{Zusammenfassung}

Acer pentaphyllum ist ein kleiner, laubabwerfender Baum aus der westchinesischen Provinz Sichuan. Am Naturstandort ist dieser Ahorn mit weniger als 200 Individuen als extrem stark gefährdet einzustufen. Innerhalb der Ahorne hat diese Art eine einzigartige, charakteristische Blattform. Die Blätter sind handförmig gefiedert und haben 5 bis 7 Fiederblättchen.

\section{Verbreitung}

Acer pentaphyllum Diels (Sapindaceae) stammt aus den 2300-2900 m hoch gelegenen Tälern der westchinesischen Provinz Sichuan. Dort ist diese Art nur noch mit 200 Individuen in vier voneinander getrennten Populationen vertreten und demnach stark bedroht. Drei dieser vier Populationen sind zudem durch Straßen- und Staudammprojekte in ihrem Fortbestand stark gefährdet (GiBBS \& CHEN 2009). Diese AhornArt wurde erst 1929 von JOSEPH ROCK auf einer von der National Geographic Society finanzierten Expedition nahe dem tibetischen Dorf Muli entdeckt und erstmals beschrieben. Am Naturstandort ist dieser Ahorn mit immergrünen Eichen, Rosen und Eschen vergesellschaftet (McNamara 2002). Das die Art sehr selten ist, ist maßgeblich auf zwei Gründe zurückzuführen. Einerseits haben die Individuen in den einzelnen isolierten Populationen untereinander nur einen sehr eingeschränkten Genpool, andererseits ist die Keimungsrate durch ungeeignete geomorphologische Standortbedingungen stark gemindert (RoH et al. 2008)

\section{2. Äußeres Erscheinungsbild und Vermehrung}

Dieser laubabwerfende Ahorn wird am Naturstandort etwa $10 \mathrm{~m}$ hoch, wächst gelegentlich auch großstrauchig (v. GELDEREN 1994). Er wird meist deutlich breiter als hoch und hat einen relativ kurzen Stamm. Häufig verzweigt sich A. pentaphyllum bereits von der Basis an, wobei die Hauptäste aufrecht orientiert sind, die Sei- tenäste dagegen mehr oder weniger waagerecht ausgebreitet wachsen. Die graue bis graubraune Borke ist zunächst glatt und erst im Alter längsrissig oder klein gefeldert. Die kleinen, spitzen Überwinterungsknospen sind dunkel- bis schwarzbraun.

Innerhalb der Gattung Acer ist die Blattform von $A$. pentaphyllum einzigartig. Die oberseits frischgrünen und unterseits bläulichen, bis $15 \mathrm{~cm}$ langen, gegenständig angeordneten Blätter sind so tief eingeschnitten, dass die Blattlappen zu einzelnen Fiedern werden. Die meisten Blätter setzen sich aus 5 (gelegentlich 7) Einzelblättchen zusammen, worauf sich der Artbeiname „pentaphyllum “ bezieht. Diese sind $8-10 \mathrm{~cm}$ lang und $0,5-1 \mathrm{~cm}$ breit. Die Blätter jüngerer Individuen haben einen auffällig grob gezähnten Rand, bei älteren Bäumen sind sie ganzrandig. Die Blattform ähnelt derjenigen des Mönchspfeffers (Vitex agnus-castus). Der bis zu $5 \mathrm{~cm}$ lange Blattstiel ist sonnenseits gerötet, ansonsten hellgrün. Die Herbstfärbung reicht von goldgelb bis hin zu leuchtend karminrot.

Im Mai erscheinen die gelblichen Blüten in terminalen und axillaren, doldentraubigen, bis 16-blütigen Blütenständen. Die Einzelblüten haben 5 Kelch-, 5 Kron- und 8 Staubblätter (DE JONG 1976). In den zwei Staubblattkreisen fehlen zwei Staubblätter, da bereits zur Blütezeit die Flügel an den Fruchtknoten so stark entwickelt sind, dass der Platz für die Staubblätter an diesen Stellen fehlt. Acer pentaphyllum wird von Insekten bestäubt. Aus den Blüten entwickeln sich die typischen Spaltfrüchte. Diese sind zu- 

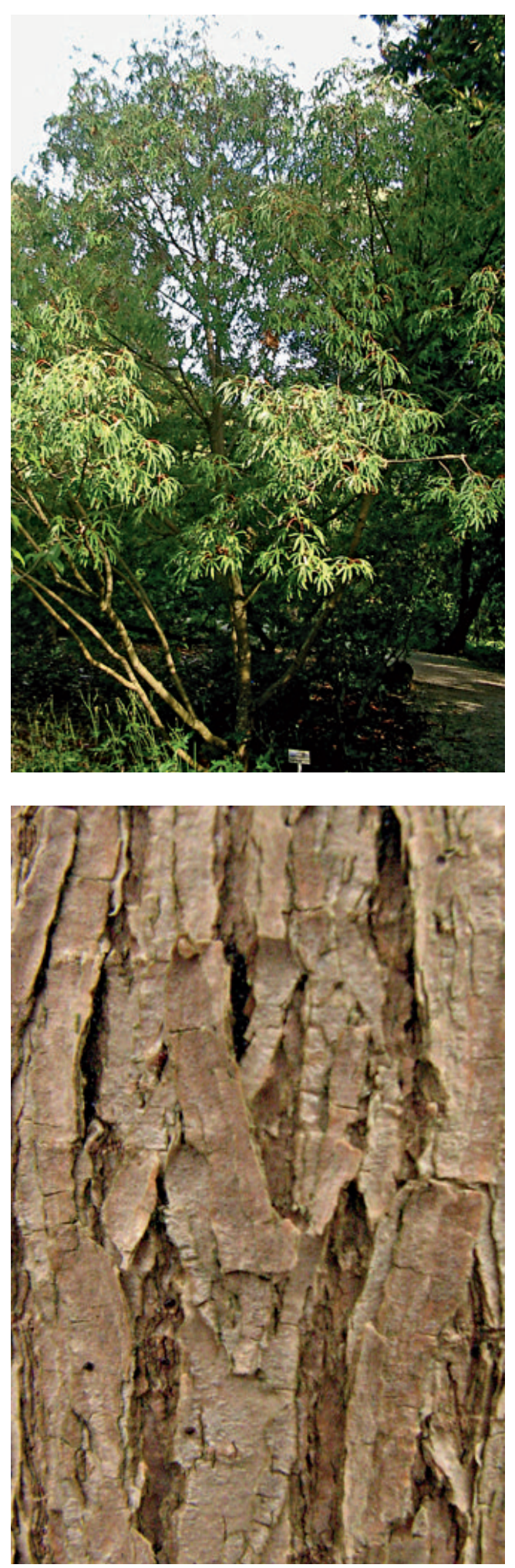

nächst grün, später jedoch braun gefärbt. Die rund $2-3 \mathrm{~cm}$ langen Fruchtflügel stehen in einem stumpfen Winkel zueinander. Die parthenokarpische Tendenz ist hoch, viele Früchte sind daher taub. In Baumschulen wird Acer pentaphyllum meist vegetativ durch Stecklinge oder Veredelungen vermehrt.

\section{Kultur}

Acer pentaphyllum ist im Aceretum der Baumschule Esveld (Boskoop, Niederlande) zu finden. Gelegentlich wird er auch in den Sortimentskatalogen niederländischer Spezialanbieter angeboten, in Mitteleuropa ist die Art allerdings als bedingt frosthart zu bezeichnen und sollte nur in der milden Winterhärtezone $8\left(-15\right.$ bis $\left.-8^{\circ} \mathrm{C}\right)$ gepflanzt werden.

In Deutschland ist dieser Ahorn nur sehr selten in Kultur. Im Freiland des Botanischen Gartens Frankfurt wird ein Individuum seit einigen Jahren erfolgreich kultiviert und gedeiht dort rechts vom Bienenhaus vor der großen Zeder. Der Baum wurde 1998 bei der Baumschule ESVELD als Containerware $(30 / 40 \mathrm{~cm})$ gekauft. Zunächst wurde der Baum im Container wintertags im Kalthaus kultiviert, im Sommer stand er draußen. Im Jahr 2006 wurde er schließlich aus Platzgründen ausgepflanzt. Obwohl Acer pentaphyllum in Deutschland nur in den Weinbauklimaten als möglicherweise ausreichend frosthart zu bezeichnen ist und daher grundsätzlich nur für die Kübelkultur empfohlen wird, ist es erstaunlich, dass das im Botanischen Garten Frankfurt ausgepflanzte Exemplar die letzten Winter überstanden hat. Zwar sind in den Wintern seine Zweige immer leicht zurückgefroren und ist der Baum von der Rotpustelkrankheit (verursacht durch den Pilz Nectria cinnabarina) befallen, doch ist er nach dem Zurückschneiden stets gut ausgetrieben. Auch den Extremwinter 2009/2010 hat er überlebt.

Außerhalb des natürlichen Standortes sind wohl die Individuen im Strybing Arboretum in San Francisco die ältesten und eindrucksvollsten.

Abb. 1 (oben): Habitus von Acer pentaphyllum.

Abb. 2 (unten): Borke. 


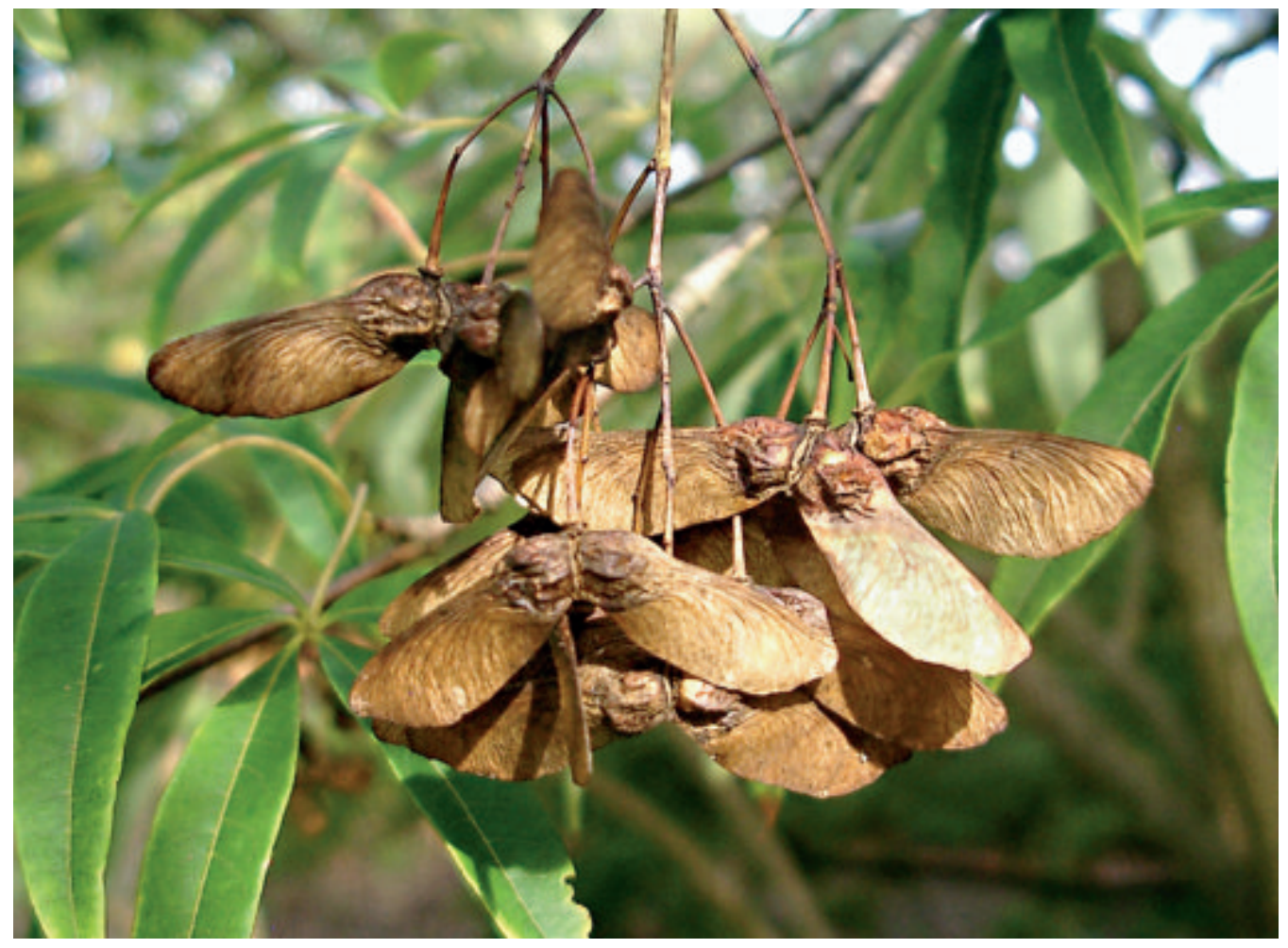

In Quarryhill Botanical Gardens, im klimatisch begünstigten Weinbauklima von Sonoma Valley, ungefähr eine Autostunde von San Francisco entfernt gelegen, befindet sich eine der größten Schutzsammlungen für Acer pentaphyllum außerhalb Chinas.

\section{Literatur}

JONG DE, P. C. 1976: Flowering and sex expression in Acer L., a biosystematic study. -

Meded. Landbouwhogschool 76: 1-201.

Gelderen van, D. M., De Jong, P. C. \&

Oterdoom, H. J. 1994: Maples of the world. - Portland. GibBS, D. \& CHEN, Y. 2009: The red list of maples. - Kew. MCNAMARA, W. A. 2002: Making a last stand: Acer pentaphyllum. - Pacific Horticulture 63: 2. Roh, M. S., McNamara, W. A., Picton, D. \& YIN, K. 2008: Assessment of genetic variation in Acer pentaphyllum based on amplified fragment length polymorphisms. J. Hortic. Sci. Biotechnol. 83: 725-731.

Abb. 3 (oben): Früchte von Acer pentaphyllum.

Abb. 4 (unten): Das Blatt erinnert an den Mönchspfeffer.

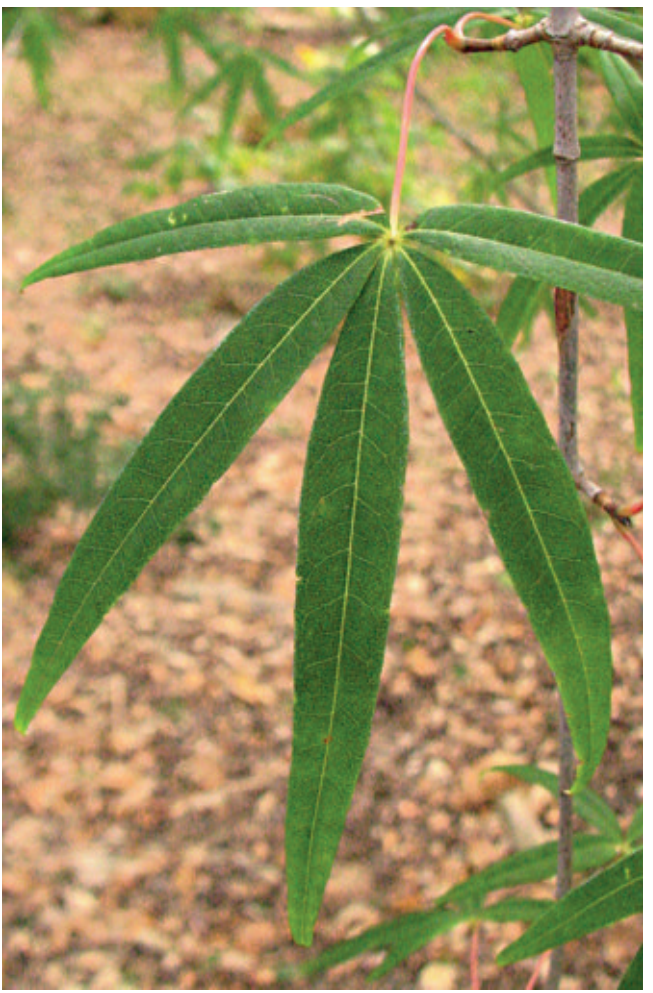

